\title{
Antithrombin supplementation during extracorporeal membrane oxygenation: study protocol for a pilot randomized clinical trial
}

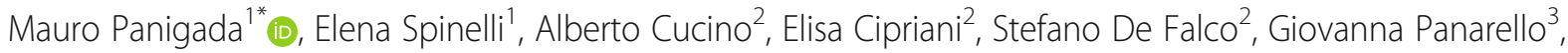 \\ Giovanna Occhipinti ${ }^{3}$, Antonio Arcadipane ${ }^{3}$, Gabriele Sales ${ }^{4}$, Vito Fanelli ${ }^{4}$, Luca Brazzi ${ }^{4}$, Cristina Novembrino ${ }^{5}$, \\ Dario Consonni ${ }^{6}$, Antonio Pesenti ${ }^{1,2}$ and Giacomo Grasselli, ${ }^{1,2}$
}

\begin{abstract}
Background: Normal levels of plasma antithrombin (AT) activity might decrease heparin requirements to achieve an adequate level of anticoagulation during treatment with extracorporeal membrane oxygenation (ECMO). Acquired AT deficiency during ECMO is common, but formal recommendations on target, timing, and rate of AT supplementation are lacking. Thus, we conceived a pilot trial to evaluate the feasibility and safety of prolonged AT supplementation in patients requiring veno-venous ECMO for respiratory failure.

Methods: Grifols Antithrombin Research Awards (GATRA) is a prospective, randomized, single blinded, multicenter, controlled two-arm trial. Patients undergoing veno-venous ECMO will be randomized to either receive AT supplementation to maintain a functional AT level between 80 and 120\% (AT supplementation group) or not (control group) for the entire ECMO course. In both study groups, anticoagulation will be provided with unfractionated heparin following a standardized protocol. The primary endpoint will be the dose of heparin required to maintain the ratio of activated partial thromboplastin time between 1.5 and 2 . Secondary endpoints will be the adequacy of anticoagulation and the incidence of hemorrhagic and thrombotic complications.

Discussion: GATRA is a pilot trial that will test the efficacy of a protocol of AT supplementation in decreasing the heparin dose and improving anticoagulation adequacy during ECMO. If positive, it might provide the basis for a future larger trial aimed at verifying the impact of AT supplementation on a composite outcome endpoint including hemorrhagic events, transfusion requirements, and mortality.
\end{abstract}

Trial registration: ClinicalTrials.gov, NCT03208270. Registered on 5 July 2017.

\section{Background}

Extracorporeal membrane oxygenation (ECMO) is a temporary life support method for patients with severe acute respiratory failure refractory to conventional treatment, and its use is continuously increasing worldwide [1]. Since exposure of blood to the non-biologic surface of the extracorporeal circuit induces a pro-thrombotic state and an inflammatory response, the use of ECMO

\footnotetext{
* Correspondence: mauro.panigada@outlook.com

${ }^{1}$ Department of Anesthesia, Critical Care and Emergency, Fondazione IRCCS

Ca' Granda Ospedale Maggiore Policlinico, Milan, Italy

Full list of author information is available at the end of the article
}

necessitates the maintenance of hemostatic balance to minimize the risk of both hemorrhagic and thrombotic complications [2]. Consequently, to avoid clotting in the extracorporeal circuit and in the patient, anticoagulation is necessary, but it increases the risk of bleeding [3]. A recent retrospective analysis on more than 2000 patients reported bleeding and thrombotic complications with a frequency of up to $45 \%$ and $60 \%$, respectively, with major impact on outcome [4].

Anticoagulation management during ECMO is usually based on continuous infusion of unfractionated heparin $[5,6]$. The heparin effect is strictly dependent on

(C) The Author(s). 2019 Open Access This article is distributed under the terms of the Creative Commons Attribution 4.0 International License (http://creativecommons.org/licenses/by/4.0/), which permits unrestricted use, distribution, and 
antithrombin (AT) activity in plasma [7, 8]. Acquired AT deficiency during ECMO is common and multifactorial [9]: possible mechanisms include consumption due to activated coagulation and long-term anticoagulation, but also impaired synthesis, degradation by elastase from activated neutrophils, and disseminated intravascular coagulation. AT deficiency contributes to heparin resistance, with resulting difficulty in achieving therapeutic anticoagulation and increased heparin dose [7]. Theoretically, normalization of AT levels should decrease heparin requirements to achieve a proper anticoagulation target [9]. This may have a relevant clinical impact because risk of bleeding during ECMO is reasonably associated with higher heparin dosage, and a better control of anticoagulation may improve patients' outcome [10]. However, formal recommendations on target, timing, and rate of AT supplementation during ECMO are lacking.

Given this lack of current knowledge, we designed a prospective randomized controlled clinical trial to evaluate the effects of a protocol of AT supplementation to achieve and maintain a normal AT activity on heparin dose, level of anticoagulation, bleeding, and thrombotic complications in adult patients undergoing ECMO for respiratory failure.

The results of this study will clarify some of the unanswered issues on AT supplementation during ECMO and will eventually provide the basis for a subsequent larger study on outcome.

\section{Methods}

\section{Study design}

The Grifols Antithrombin Research Awards (GATRA) study is a pilot, prospective, randomized, single blinded, multicenter, controlled two-arm trial that will be performed on adult patients undergoing veno-venous ECMO for severe respiratory failure. The study will be conducted in adherence to the principles of the World Medical Association's Declaration of Helsinki and in accordance with the Medical Research Involving Human Subjects Act (WMO). The Ethics Committee of the coordinating center (Comitato Etico Milano Area B) approved the protocol on April 18, 2017 (Version 1.6.7 - April 4, 2017), and approval of the local Ethics Committees will be required before starting the trial in the participating centers. The study was authorized by AIFA (Agenzia Italiana del Farmaco) on April 11, 2017 (EudraCT number 2016-004534-23) and registered at www.ClinicalTrials.gov with code NCT03208270. Informed consent to participate in the clinical trial will be obtained, and information on the clinical trial will be given to each patient. If the patient is not capable of giving informed consent at the time of enrollment, a deferred consent will be given. The informed consent will be sought by the investigator from the patient, and the information about the study will be given to the patient or his/her legally designated representative as soon as possible. If the patient does not consent to the study, he/she will be informed of the right to object to the use of data obtained from the clinical trial. The Standard Protocol Items: Recommendations for Interventional Trials (SPIRIT) checklist is provided in Additional file 1, and the SPIRIT figure is included in the main body of the manuscript (Fig. 1).

\section{Setting}

The GATRA trial will be performed in the intensive care units (ICUs) of Italian ECMO referral centers. The coordinating center will be Fondazione IRCCS $\mathrm{Ca}^{\prime}$ Granda OspedaleMaggiore Policlinico in Milan, Italy.

\section{Study population}

Consecutive adult patients admitted to the participating centers requiring veno-venous ECMO as a support for respiratory failure will be enrolled in the study. Exclusion criteria will be pre-existing heparin-induced thrombocytopenia or other contraindications to heparin use (namely high risk of bleeding after major surgery prompting the decision to start ECMO without systemic anticoagulation). The Consolidated Standards of Reporting Trials (CONSORT) diagram of the GATRA trial is presented in Fig. 2.

\section{Randomization}

As soon as the clinical decision to initiate ECMO is made, the patient will be enrolled in the study. Patients will be block randomized per center through an online automatic centralized and computerized system to one of the two groups (1:1 ratio): AT supplementation group or control group.

\section{Interventions \\ Antithrombin supplementation group}

Patients randomized to the AT supplementation group will receive supplementation of AT concentrate to maintain a functional AT level between 80 and $120 \%$. The AT level will be measured prior to ECMO start and then once daily and supplementation adjusted accordingly. In the study group, AT concentrate supplementation will be interrupted when AT levels exceeds $120 \%$ and resumed as soon as the levels drop below $80 \%$. The protocol for AT supplementation is illustrated in Fig. 3.

\section{Control group}

Patients randomized to the control group will not receive supplementation of AT unless heparin resistance occurs. The following safety criteria will be applied to define "heparin resistance": when a patient in the control group requires more than $35 \mathrm{IU} / \mathrm{kg} / \mathrm{h}$ of heparin and 


\begin{tabular}{|c|c|c|c|c|}
\hline & \multicolumn{4}{|c|}{ STUDY PERIOD } \\
\hline & Enrolment & Allocation & Post-allocation & Close-out \\
\hline TIMEPOINT $^{\star *}$ & $\begin{array}{c}\text { Before ECMO } \\
\text { start }\end{array}$ & $\begin{array}{l}\text { ECMO } \\
\text { start }\end{array}$ & $\begin{array}{l}\text { Daily for the } \\
\text { entire ECMO } \\
\text { duration }\end{array}$ & $\begin{array}{c}I C U \\
\text { discharge/death }\end{array}$ \\
\hline \multicolumn{5}{|l|}{ ENROLMENT: } \\
\hline \multirow{3}{*}{$\begin{array}{r}\text { Eligibility screen } \\
\text { Informed consent } \\
\text { Allocation }\end{array}$} & $X$ & & & \\
\hline & $\mathrm{X}$ & & & \\
\hline & & $\mathrm{X}$ & & \\
\hline \multicolumn{5}{|l|}{ INTERVENTIONS: } \\
\hline \multicolumn{5}{|l|}{$\begin{array}{r}\text { Antithrombin } \\
\text { supplementation group }\end{array}$} \\
\hline \multicolumn{5}{|l|}{ Control group } \\
\hline \multicolumn{5}{|l|}{ ASSESSMENTS: } \\
\hline \multirow{3}{*}{$\begin{array}{r}\text { Screening test for } \\
\text { thrombofilia } \\
\text { Coagulation and ECMO } \\
\text { parameters } \\
\text { Heparin dose, transfusions, } \\
\text { bleeding and thrombotic } \\
\text { events }\end{array}$} & & $\mathrm{X}$ & $X$ & \\
\hline & & & $\mathrm{X}$ & \\
\hline & & & $x$ & \\
\hline $\begin{array}{r}\text { ECMO duration, ICU LOS } \\
\text { and 28-day survival }\end{array}$ & & & & $\mathrm{X}$ \\
\hline
\end{tabular}

Fig. 1 Intervention and assessment schedule

his/her AT plasma level is equal to or lower than $50 \%$. Dosage regimen for AT supplementation in the control group will follow the same supplementation algorithm as in the treatment group. AT will be supplemented to achieve an AT plasma level higher than 50\%. The AT level will be measured prior to ECMO start and then once daily.

\section{Anticoagulation protocol for both groups}

In both study groups, anticoagulation during ECMO will be provided with unfractionated heparin following the institutional protocol of the coordinating center, as previously published [10] (Fig. 4). Briefly, a heparin bolus of 50-70 IU $/ \mathrm{kg}$, depending on baseline activated partial thromboplastin time (aPTT) value, will be administered at ECMO start, followed by $18 \mathrm{IU} / \mathrm{kg} / \mathrm{h}$ continuous infusion. For the first $12 \mathrm{~h}$ after ECMO start, anticoagulation will be monitored with activated clotting time (ACT) (therapeutic range $180-210 \mathrm{~s}$, performed every 1 or $2 \mathrm{~h}$ according to the standardized protocol). From the 13th hour on, anticoagulation will be guided by aPTT, with a target aPTT ratio (patient-to-normal) range of 1.5-2.0. The frequency of aPTT measurements may vary from a minimum of three times per day to a maximum of six depending on the standardized protocol. When the
aPTT value falls well below the desired range, a bolus of heparin will be administered. When the value is too high, infusion will be stopped for either 30 or $60 \mathrm{~min}$. In both groups the following safety criteria will be used: in case of major surgery, during the first $24 \mathrm{~h}$ after operation, minimal levels of anticoagulation will be allowed to minimize the risk of bleeding. In case of minor bleeding (i.e., dripping from the site of the vascular catheter's cannulation), physicians shall maintain anticoagulation targeted to the lower level (i.e., aPTT ratio 1.5). In case of clinically significant bleeding, heparin infusion may be interrupted until bleeding ceases; infusion will be restored starting at 50$70 \%$ of the pre-existing dose. No changes will be made to the AT dosage regimen.

\section{Criteria for transfusions of blood products and replacement of the ECMO circuit}

For the entire duration of the study, hemoglobin will be maintained at $>10 \mathrm{~g} / \mathrm{dL}$, platelet count $>50,000 / \mathrm{mm}^{3}$, and fibrinogen $>150 \mathrm{mg} / \mathrm{dL}$, through packed red blood cells, platelets, and fresh frozen plasma and/or fibrinogen concentrate transfusions, respectively.

To standardize the clinical decision to replace the ECMO circuit with a new one, an ad hoc "circuit 


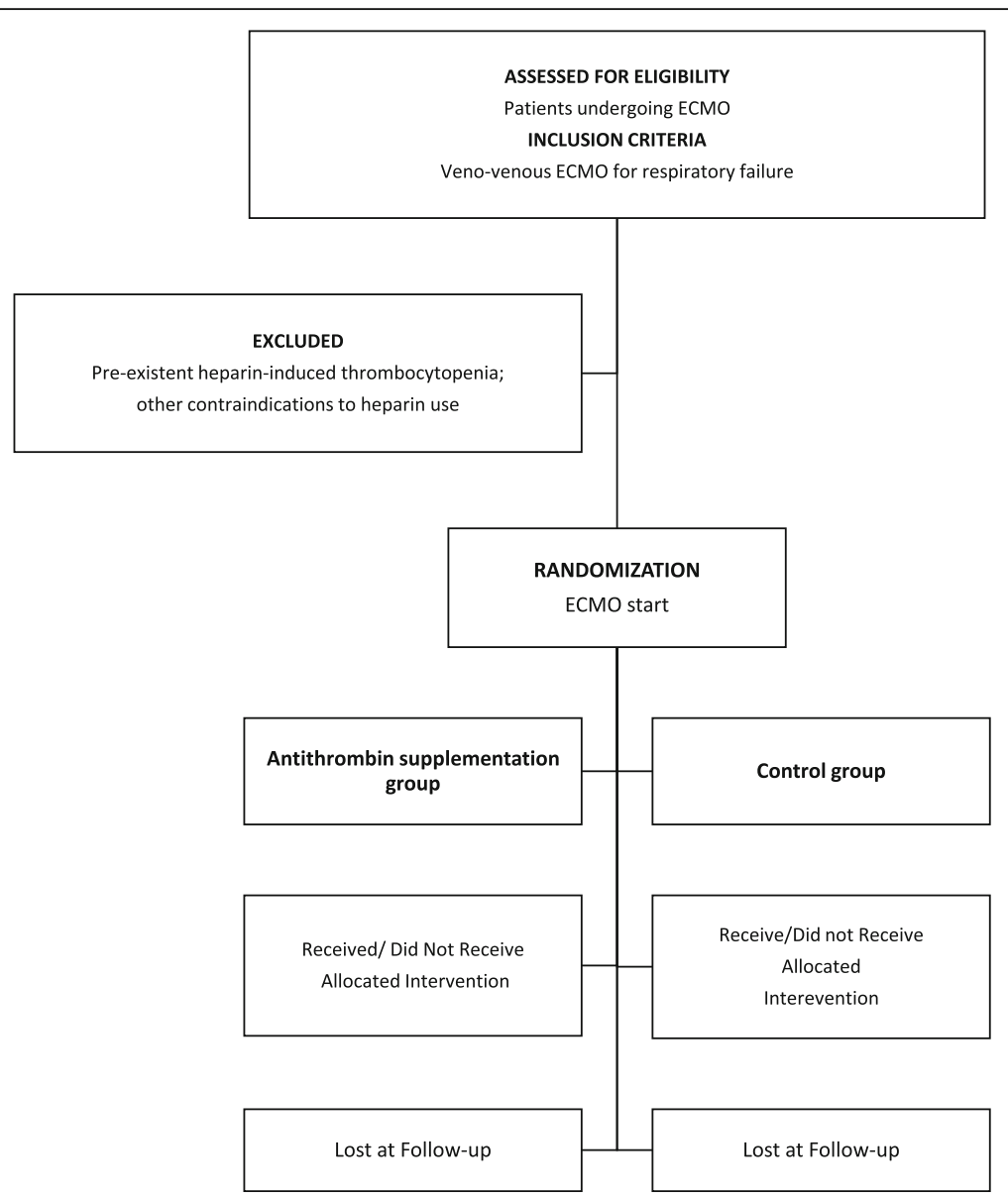

Fig. 2 CONSORT flow diagram

change-out score" was developed that considers the performance of the oxygenator, signs of coagulation activation by the extracorporeal circuit (e.g., drop in platelet count, increase in D-dimer level, and decrease in fibrinogen level), and the presence of hemolysis. The "circuit change-out score" includes two different conditions (A and $B$ ) defined by different sets of criteria. Condition A requires all the following three criteria to be satisfied: decrease in platelet count greater than $50 \%$ in $48 \mathrm{~h}$; decrease in fibrinogen level greater than $33 \%$ in $48 \mathrm{~h}$; increase in D-dimer level greater than 100\% with D-dimer level higher than $30,000 \mathrm{ng} / \mathrm{mL}$. Condition B requires two of the following three criteria to be satisfied: platelet count lower than $50,000 / \mathrm{mL}$; fibrinogen lower than 150 $\mathrm{mg} / \mathrm{dL}$; D-dimer higher than $50,000 \mathrm{ng} / \mathrm{mL}$, provided that $\mathrm{D}$-dimer elevation is one of the two "B" conditions.

\section{Stopping rules}

Patients will be supplemented with AT during ECMO according to the assigned group and followed up for the entire duration of ECMO. AT supplementation will be stopped at ECMO discontinuation.
Premature discontinuation of the study will be allowed when one of the following conditions occurs: (1) death; (2) onset of heparin-induced thrombocytopenia requiring shift to an alternative anticoagulant drug to heparin; (3) unexpected circumstances that prevent proper heparin or AT supplementation.

\section{End of follow-up}

Enrolled patients will be observed until ICU discharge or death, whichever comes first.

\section{Study endpoints \\ Primary endpoint}

The primary endpoint will be the amount of heparin infused to each patient, calculated as the total administered heparin per kilogram of body weight (including boluses) divided by the total duration of heparin infusion in hours (IU/kg/h).

\section{Secondary endpoints}

Adequacy of anticoagulation will be assessed through anti-factor Xa levels. 
Daily measurement Of AT activity

$\rightarrow$ Antithrombin MAINTENANCE dose

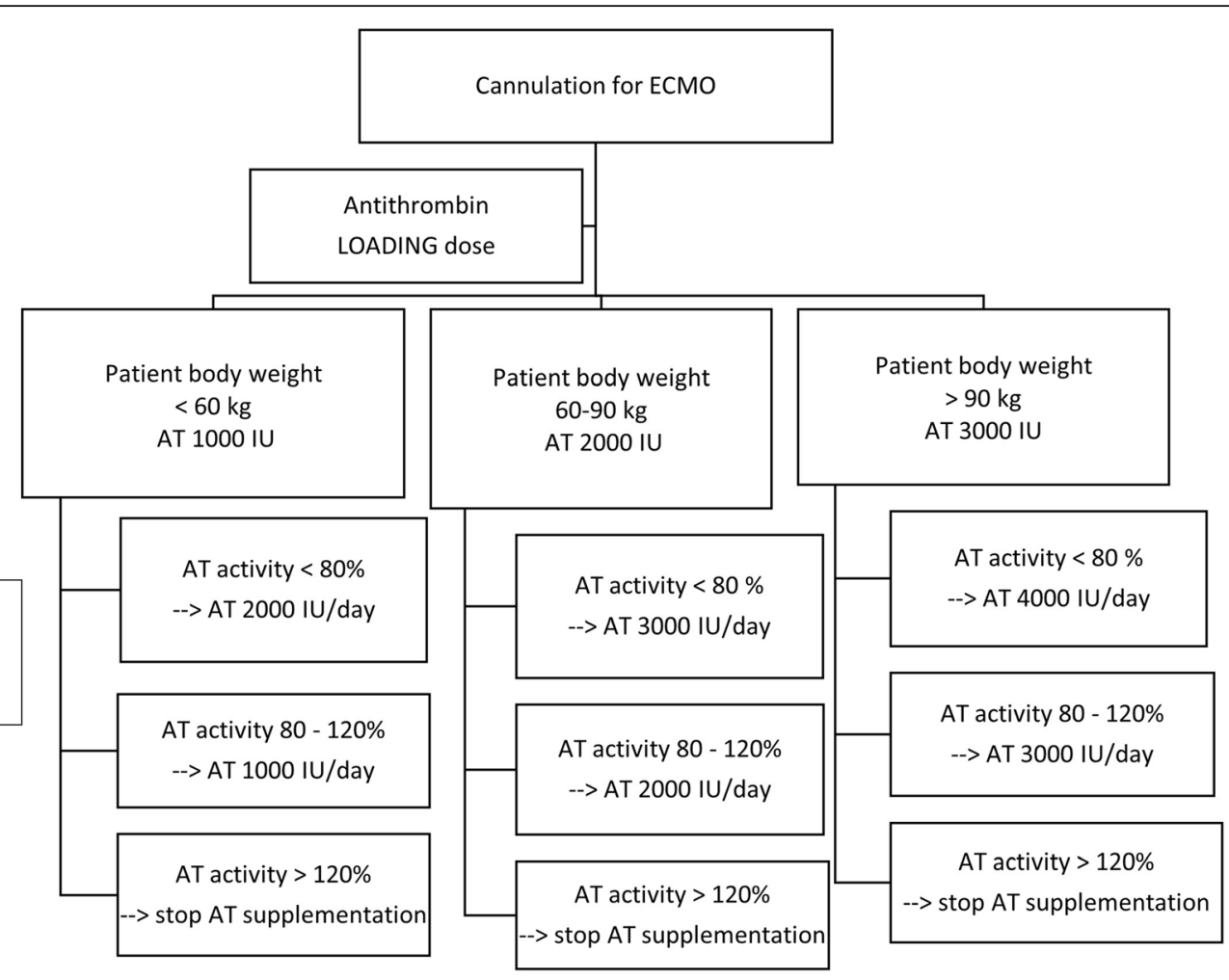

Fig. 3 Protocol for anthithrombin supplementation

\begin{tabular}{|c|c|c|c|c|c|c|c|}
\hline \multicolumn{8}{|c|}{ UFH adjustment algorithm for the first $12 \mathrm{~h}$ of ECMO support (using ACT measurement) } \\
\hline ACT (sec) & $<140$ & $141-160$ & $161-179$ & $180-210$ & 211-240 & 241-270 & $>\mathbf{2 7 0}$ \\
\hline UFH BOLUS (IU/Kg) & 20 & 10 & - & - & - & - & - \\
\hline UFH infusion stop (min) & - & - & - & - & - & 30 & 60 \\
\hline UFH infusion adjustment & $+30 \%$ & $+20 \%$ & $+10 \%$ & - & $-10 \%$ & $-20 \%$ & $-30 \%$ \\
\hline Time to next control & 1h & 1h & $2 \mathrm{~h}$ & $2 \mathrm{~h}$ & $2 \mathrm{~h}$ & 1h & 1h \\
\hline \multicolumn{8}{|c|}{ UFH adjustment algorithm after the first $12 \mathrm{~h}$ of ECMO (using aPTT ratio) } \\
\hline aPTT (ratio) & $<1,2$ & $1,21-1,30$ & $1,31-1,49$ & $1,5-2$ & $2,01-2,25$ & $2,26-2,49$ & $>2,5$ \\
\hline UFH BOLUS (IU/Kg) & 20 & 10 & - & - & - & - & - \\
\hline UFH infusion stop (min) & - & - & - & - & - & 30 & 60 \\
\hline UFH infusion adjustment & $+20 \%$ & $+15 \%$ & $+10 \%$ & - & $-10 \%$ & $-20 \%$ & $-30 \%$ \\
\hline Time to next control & $4 h$ & $4 \mathrm{~h}$ & $6 h$ & $8 \mathrm{~h}$ & $6 h$ & $4 h$ & $2 \mathrm{~h}$ \\
\hline
\end{tabular}

Fig. 4 Anticoagulation protocol 
The study will also provide a preliminary evaluation of the safety of AT supplementation by comparing the incidence of the following adverse events in the two study groups: bleeding complications, classified according to a modified version of the Bleeding Academic Research Consortium score to our clinical settings [11, 12]; blood products transfusion requirements; patient's or circuit thrombosis.

\section{Data collection}

At enrollment, we will anonymously collect patients' demographic information (e.g., age, sex, height, weight), past (e.g., chronic medications including anticoagulant and antiplatelet drugs) and recent (e.g., etiology of the acute respiratory failure) medical history, and ECMO configuration with cannulation sites. A blood sample will be drawn from patients to test inherited or acquired thrombophilia (proteins C and S, Factor (F) V G1691A and FII G20210A, lupus anticoagulant, anti-cardiolipin, and anti- $\beta 2$-glycoprotein 1 antibodies).

The following data will be recorded every day for the whole study duration: daily heparin dosage; coagulation parameters (including D-dimer, fibrinogen, aPTT ratio, prothrombin time (PT) ratio, anti-factor Xa, antithrombin activity, ACT); complete blood count; indexes of hemolysis (hemoglobin, free hemoglobin, and haptoglobin levels); transfusion of blood products.

Bleeding and thrombotic events will also be recorded. Within $24 \mathrm{~h}$ after ECMO removal, a Doppler ultrasonography of the cannulated vessels and of the vena cava will be performed to exclude thrombosis due to vascular cannulation. Functional ECMO parameters will be evaluated once per day (membrane oxygenator shunt, blood flow resistance, oxygenation performance) and recorded.

Evaluation of clinical outcome will include duration of ECMO, ICU length of stay, and survival at 28 days.

Collected data will be entered in an electronic case report form (eCRF) available online at a dedicated website (https://ecmostudy.fbk.eu), with protected individual access for each participating center. Patient data will be anonymous and coded according to a number. The eCRF includes tools to promote data quality, such as range checks for data values. Data monitoring will be performed by means of queries on the database done by statisticians and analyzed to identify abnormalities and inconsistencies.

\section{Statistical considerations}

All statistical analyses will be done with Stata version 14.1 (StataCorp, College Station, TX, USA) in the coordinating centers at the Epidemiology Unit of Fondazione IRCCS $\mathrm{Ca}^{\prime}$ Granda Ospedale Maggiore Policlinico in Milan, Italy.

\section{Sample size}

Sample size is calculated using the confidence interval approach upon the primary outcome measure of reduction of heparin dose in the study group treated with AT to maintain a normal AT plasma level (considered to be a level higher than $80 \%$ ) compared to the control group. Preliminary data from patients in ECMO, cared for with the same heparin titration protocol of this study, showed that the mean heparin dosage was $18 \mathrm{IU} / \mathrm{kg} / \mathrm{h}$ and $13 \mathrm{IU} /$ $\mathrm{kg} / \mathrm{h}$ in patients with AT activity lower and higher than the median (73.5\%), respectively. Assuming an estimated difference of $5 \mathrm{IU} / \mathrm{kg} / \mathrm{h}$ of heparin between the two groups, a sample size of 40 patients (20 in each treatment group) would yield a $95 \%$ confidence interval of average heparin reduction from 1 to $9 \mathrm{IU} / \mathrm{kg} / \mathrm{h}$ ).

\section{Proposal for analysis}

Baseline variables will be compared between each randomized arm to assess the randomization performance and baseline balance between arms. We will not perform statistical testing for baseline comparison between arms, because any differences in baseline characteristics are consequences of chance due to randomization. All analyses will be conducted in accordance with the intention-to-treat principle and in accordance with the CONSORT statement.

For the primary and secondary outcomes, continuous variables will be reported as means \pm standard deviations or medians and $25-75 \%$ interquartile ranges. Categorical variables will be reported as absolute and relative frequencies. Differences between groups will be tested using Mann-Whitney and chi-square or Fisher's exact tests, as appropriate. Estimates of the effects and their confidence intervals will also be reported. In case of evident imbalances for key baseline variables between treated patients and controls, we will perform statistical adjustment using regression models.

For repeated outcomes within subject, the association between AT dosage, heparin dosage, bleeding, and monitoring methods will be evaluated using random-effect models.

\section{Study organization}

This is an investigator-initiated trial. Two principal investigators (MP and GG) designed the study protocol. Each participating center will indicate a local investigator in charge of the study. The principal investigators are responsible for administrative management and communication with the local investigators and for helping the participating clinical sites in trial management, record keeping, and data management. The study will be conducted with the financial support of Grifols (GATRA - Antithrombin Research Awards 2016). Funders will not have any role in the analysis and 
interpretation of the study results or in the decision to submit the report for publication. The local investigators guarantee the integrity of data collection. All adverse events will be monitored by the coordinating center and reported to AIFA according to the national legislation. Specific patient insurance will be granted to cover all unexpected adverse events caused by the study interventions.

\section{Ancillary study}

The rationale for AT supplementation during ECMO is also based on its coagulation-independent anti-inflammatory effects. Some of these mechanisms include endothelial release of prostacyclin that inhibits aggregation and activation of platelets $[13,14]$ and the reduction of various cytokines and chemokines from the endothelial cells $[15,16]$.

To evaluate markers of inflammation and epithelial and endothelial damage, 4 aliquots of plasma, $0.5 \mathrm{~mL}$ each, will be obtained from each patient in the GATRA study, by centrifugation of ethylenediaminetetraacetic acid (EDTA) blood at 3000 revolutions per minute (RPM) for $15 \mathrm{~min}$ at $4{ }^{\circ} \mathrm{C}$, prior to ECMO start, $24 \mathrm{~h}$ after ECMO start, $72 \mathrm{~h}$ after ECMO start, before ECMO removal, and 7 days after ECMO removal (or before discharge from the ICU if this happens first). Then the aliquots will be stored, shortly after collection, at $-80^{\circ} \mathrm{C}$ until levels of tumor necrosis factor (TNF)- $\alpha$, interleukin (IL)-1 $\beta$, IL-6, IL-8, IL-10, endocan, and syndecan are measured. At the same time points we will also collect blood to evaluate the transcriptional profile of circulating cells in whole blood. To identify changes in the transcriptional activation of genes involved in inflammation, epithelial, and endothelial damage, $2.5 \mathrm{~mL}$ of whole blood will be collected in RNA PAXgene tubes prior to ECMO start and 24 and $72 \mathrm{~h}$ after ECMO start. Specimens will be stored at $-20^{\circ} \mathrm{C}$ until transcriptome profiling is performed [17].

\section{Discussion}

GATRA is a pilot randomized controlled trial aimed at testing a protocol of AT supplementation in patients on ECMO for severe respiratory failure. It will assess the feasibility and the safety of prolonged AT supplementation to target normal AT levels and its efficacy in decreasing the heparin dose and improving anticoagulation adequacy. If positive, it might provide the basis for a future larger trial aimed at verifying the impact of AT supplementation on a composite outcome endpoint including hemorrhagic events, transfusion requirements, and mortality.

\section{Antithrombin supplementation during ECMO}

Heparin mainly exerts its anticoagulant effect by binding AT and facilitating the inhibition of thrombin by AT.
When heparin binds to AT, it converts AT from a slow to a rapid thrombin inhibitor. Therefore, the therapeutic activity of heparin depends on the availability of circulating AT. The heparin/AT also inhibits factor Xa and to a lesser extent other activated coagulation factors (Xa, IXa, XIa, and XIIa) [18]. Another possible anticoagulant mechanism of heparin includes the release of tissue factor pathway inhibitor from endothelial cells [19]. Although AT is needed by heparin to properly exploit its anticoagulant activity, there is no consensus on AT supplementation during ECMO. Guidelines suggest supplementing AT in ECMO only when its deficiency coexists with heparin resistance [6]. Few studies evaluated the effect of AT supplementation during ECMO without a consensus on the target level to be maintained. Small and retrospective studies in the pediatric population examined the administration of AT during ECMO but yielded inconclusive results, with some reporting no effects on heparin dose and bleeding [20-22] and others observing decreased heparin requirements [23-25] and fewer transfusions [25]. These contrasting results might be explained by the high variability in dosing schemes, target levels, timing, and duration of AT supplementation.

\section{Development of the protocol for AT supplementation: practical and operational issues}

Since dosing recommendations are not provided for acquired AT deficiency, the protocol for AT supplementation was developed following manufacturer recommendations for hereditary deficiency. Supplementation dosing is based on patient body weight and the difference between the actual and the desired AT activity. A level of AT activity corresponding to the normal range (80-120\%) was chosen as the target for supplementation. The calculated dose was rounded up to the nearest vial size, and dose tiers were created using ranges of AT activity to minimize bedside calculations. The protocol mandates, for patients in the AT supplementation group, the administration of a loading dose of AT upon ECMO cannulation. As the level of AT activity before ECMO start was expected not to be available for most patients, the loading dose is based solely on the patient body weight. Subsequent dose adjustments are based on the patient body weight and the plasma AT activity, which must be measured daily. Patients with a plasma AT activity between 80 and $120 \%$ will receive a maintenance dose, which will be increased by $1000 \mathrm{IU}$ if the plasma AT activity is lower than $80 \%$. Observational studies suggest that intermittent administration of AT is less effective than continuous infusion in correcting AT deficiency; moreover, rapid AT binding to heparin complexes after bolus administration could theoretically increase the risk for bleeding. Based on the clinical experience of our and other ECMO centers $[26,27]$, administration of AT via an 
extended infusion decreases heparin rate modifications and avoids AT levels fluctuations, without increasing the risk of major bleeding. Therefore, the protocol prescribes AT administration by extended infusion both for loading and maintenance doses.

\section{Study endpoints}

Hemorrhagic complications remain a major cause of morbidity and mortality in ECMO patients [4]. Hence, protocols to guide anticoagulation management are key in the management of these patients. Physiological considerations suggest that normalization of AT levels might improve adequacy of heparin anticoagulation and possibly decrease the risk of bleeding. Indeed, in adult patients undergoing ECMO for respiratory failure, lower AT levels were associated with increased need for transfusion [26], and AT supplementation facilitated achievement of target anticoagulation without increasing heparin dosage [27]. A higher degree of anticoagulation [28] is associated with hemorrhagic events during ECMO, but risk of bleeding is multifactorial and does not depend solely on anticoagulation. We chose the reduction of heparin dose as the primary endpoint for this pilot trial; however, it is conceivable that larger studies will be needed to detect the hypothesized effect on clinical outcome.

\section{Study strengths and limitations}

One of the strengths of the study is that the protocol contains a well-defined heparin anticoagulation protocol, which has already been adopted in a previous multicenter trial [29]. This should facilitate its application and minimize deviations in anticoagulation management which could jeopardize the effect of AT supplementation on study endpoints. Although the decision to transfuse blood products is clinical, the protocol recommends well-defined targets for hemoglobin, platelet count, and fibrinogen. Similarly, criteria for considering replacement of the ECMO circuit are provided.

One limitation of the GATRA trial is that blinding is not possible due to the nature of the intervention. However, the factors that can potentially affect the primary outcome (in particular, heparin dose adjustments and transfusion of blood products) are regulated by the protocol.

\section{Trial status}

The GATRA trial is currently recruiting patients. The protocol was approved by the Ethics Committee of the coordinating center on April 18, 2017. The first patient was enrolled on August 8, 2017. As of September 9 2018, 36 patients have been screened and 23 patients have been randomized (11 interventional group, 12 control group) in the coordinating center. Recruitment is expected to be completed in May 2019.

\section{Additional file}

Additional file 1: SPIRIT 2013 checklist. (DOC $121 \mathrm{~kb}$ )

\begin{abstract}
Abbreviations
ACT: Activated clotting time; AlFA: Agenzia Italiana del Farmaco; aPTT: Activated partial thromboplastin time; AT: Antithrombin;

ECMO: Extracorporeal membrane oxygenation; eCRF: Electronic case report form; EDTA: Ethylenediaminetetraacetic acid; ICl: Intensive care unit; IL: Interleukin; IU: International Unit; OR: Odds ratio; PT: Prothrombin time; RPM: Revolutions per minute; TNF: Tumor necrosis factor
\end{abstract}

\section{Acknowledgements \\ None.}

\section{Funding}

The study is funded with the Grifols ${ }^{\circledast}$ Antithrombin Research Award (GATRA) 2016.

\section{Availability of data and materials}

Collected data will be entered in an eCRF available online at a dedicated website (https://ecmostudy.fbk.eu), with protected individual access for each participating center. The datasets used and/or analyzed during the current study will be available from the corresponding author on reasonable request.

\section{Authors' contributions}

MP and GG conceived the study and wrote the study protocol. AP and ES reviewed the study protocol for important intellectual content. All authors reviewed the study protocol and approved the final version. MP, ES, AC, EC, SDF, GP, GO, AA, GS, VF, and LB collaborated in enrollment, treatment, and data collection in the first patients. CN performed laboratory analyses. DC provided support for the statistical analysis. ES and MP drafted the present manuscript. All authors reviewed the present manuscript and approved the final version.

\section{Ethics approval and consent to participate}

Central ethical approval has been confirmed from the Milan Area B Ethics Committee (ref. approval no. 191_2017bis), and we will not begin recruiting at other centers in the trial until local ethical approval has been obtained. Informed consent will be obtained from all the patients enrolled following local regulations.

\section{Consent for publication}

Not applicable.

\section{Competing interests}

MP was awarded GATRA Grifols Antithrombin Research Awards in 2016. He received payment and travel support for lectures from Shire ${ }^{\circledast}$ and Werfen ${ }^{\circledast}$ Instrumentation Laboratory. GG received payment for lectures from Pfizer, Getinge, Draeger, and Fisher \& Paykel and support for travel/congress activities from Biotest and Getinge (all unrelated to the present manuscript). The other authors declare that they have no competing interests.

\section{Publisher's Note}

Springer Nature remains neutral with regard to jurisdictional claims in published maps and institutional affiliations.

\section{Author details}

${ }^{1}$ Department of Anesthesia, Critical Care and Emergency, Fondazione IRCCS Ca' Granda Ospedale Maggiore Policlinico, Milan, Italy. ${ }^{2}$ Department of Pathophysiology and Transplantation, University of Milan, Milan, Italy. ${ }^{3}$ Department of Anesthesiology and Intensive Care, ISMETT IRCCS (Istituto Mediterraneo per i Trapianti e Terapie ad Alta Specializzazione), UPMC, Palermo, Italy. ${ }^{4}$ Department of Surgical Sciences, University of Turin, Turin, 
Italy. ${ }^{5}$ Clinical Laboratory, Fondazione IRCCS Ca' Granda Ospedale Maggiore Policlinico, Milan, Italy. 'Epidemiology Unit, Fondazione IRCCS Ca' Granda Ospedale Maggiore Policlinico, Milan, Italy.

Received: 1 December 2018 Accepted: 29 April 2019

Published online: 11 June 2019

\section{References}

1. Combes A, Brodie D, Bartlett R, Brochard L, Brower R, Conrad S, et al. Position paper for the organization of extracorporeal membrane oxygenation programs for acute respiratory failure in adult patients. Am J Respir Crit Care Med. 2014;190(5):488-96.

2. Muntean W. Coagulation and anticoagulation in extracorporeal membrane oxygenation. Artif Organs. 1999;23(11):979-83.

3. Annich $G$, Adachi I. Anticoagulation for pediatric mechanical circulatory support. Pediatr Crit Care Med. 2013;14:S37-42.

4. Dalton HJ, Garcia-Filion P, Holubkov R, Moler FW, Shanley T, Heidemann S, et al. Association of bleeding and thrombosis with outcome in extracorporeal life support. Pediatr Crit Care Med. 2015;16(2):167-74.

5. Oliver WC. Anticoagulation and coagulation management for ECMO. Semin Cardiothorac Vasc Anesth. 2009;13(3):154-75.

6. ELSO Anticoagulation Guideline - 2014. https://www.elso.org/Portals/0/Files/ elsoanticoagulationguideline8-2014-table-contents.pdf.

7. Opal SM, Kessler CM, Roemisch J, Knaub S. Antithrombin, heparin, and heparan sulfate. Crit Care Med. 2002;30(5 Suppl):S325-31.

8. Pratt CW, Church FC. Antithrombin: structure and function. Semin Hematol. 1991;28(1):3-9

9. Maclean PS, Tait RC. Hereditary and acquired antithrombin deficiency: epidemiology, pathogenesis and treatment options. Drugs. 2007;67(10): 1429-40.

10. Protti A, LAcqua C, Panigada M. The delicate balance between pro-(risk of thrombosis) and anti-(risk of bleeding) coagulation during extracorporeal membrane oxygenation. Ann Transl Med. 2016;4(7):139.

11. Mehran R, Rao SV, Bhatt DL, Gibson CM, Caixeta A, Eikelboom J, et al. Standardized bleeding definitions for cardiovascular clinical trials: a consensus report from the Bleeding Academic Research Consortium. Circulation. 2011:123(23):2736-47.

12. Panigada M, lapichino G, L'Acqua C, Protti A, Cressoni M, Consonni D, et al. Prevalence of "flat-line" thromboelastography during extracorporeal membrane oxygenation for respiratory failure in adults. ASAIO J. 2016:62(3):302-9.

13. Uchiba M, Okajima K, Murakami K, Okabe H, Takatsuki K. Effects of antithrombin III (AT III) and Trp49-modified AT III on plasma level of 6-ketoPGF1 alpha in rats. Thromb Res. 1995;80(3):201-8.

14. Roemisch J, Gray E, Hoffmann JN, Wiedermann CJ. Antithrombin: a new look at the actions of a serine protease inhibitor. Blood Coagul Fibrinolysis. 2002;13(8):657-70.

15. Wang J, Wang Y, Wang J, Gao J, Tong C, Manithody C, et al. Antithrombin is protective against myocardial ischemia and reperfusion injury. J Thromb Haemost. 2013;11(6):1020-8.

16. Isik S, Tuncyurek P, Zengin NI, Demirbag AE, Atalay F, Yilmaz S, et al. Antithrombin prevents apoptosis by regulating inflammation in the liver in a model of cold ischemia/warm reperfusion injury. Hepatogastroenterology. 2012:59(114):453-7.

17. dos Santos CC, Okutani D, Hu P, Han B, Crimi E, He X, et al. Differential gene profiling in acute lung injury identifies injury-specific gene expression. Crit Care Med. 2008:36:855-65.

18. Hirsh J, Raschke R. Heparin and low-molecular-weight heparin: the Seventh ACCP Conference on Antithrombotic and Thrombolytic Therapy. Chest. 2004;126:1885-2035

19. Kemme MJB, Burggraaf J, Schoemaker RC, Kluft C, Cohen AF. Quantification of heparin-induced TFPI release: a maximum release at low heparin dose. $\mathrm{Br}$ J Clin Pharmacol. 2002;54(6):627-34.

20. Wong TE, Delaney M, Gernsheimer T, Matthews DC, Brogan TV, Mazor R, et al. Antithrombin concentrates use in children on extracorporeal membrane oxygenation: a retrospective cohort study. Pediatr Crit Care Med. 2015;16: 264-9.

21. Niebler RA, Christensen M, Berens R, Wellner H, Mikhailov T, Tweddell JS. Antithrombin replacement during extracorporeal membrane oxygenation. Artif Organs. 2011;35(11):1024-8.
22. Byrnes JW, Swearingen CJ, Prodhan P, Fiser R, Dyamenahalli U. Antithrombin III supplementation on extracorporeal membrane oxygenation: impact on heparin dose and circuit life. ASAIO J. 2014;60(1): $57-62$

23. Nelson KM, Hansen LA, Steiner ME, Fischer GA, Dehnel J, Gupta S. Continuous antithrombin III administration in pediatric veno-arterial extracorporeal membrane oxygenation. J Pediatr Pharmacol Therapeutics. 2017;22:266-71.

24. Ryerson LM, Bruce AK, Lequier L, Kuhle S, Massicotte MP, Bauman ME. Administration of antithrombin concentrate in infants and children on extracorporeal life support improves anticoagulation efficacy. ASAIO J. 2014;60(5):559-63.

25. Perry R, Stein J, Young G, Ramanathan R, Seri I, Klee L, et al. Antithrombin III administration in neonates with congenital diaphragmatic hernia during the first three days of extracorporeal membrane oxygenation. J Pediatr Surg. 2013;48(9):1837-42.

26. Martucci G, Panarello G, Occhipinti G, Ferrazza V, Tuzzolino F, Bellavia D, et al. Anticoagulation and transfusions management in veno-venous extracorporeal membrane oxygenation for acute respiratory distress syndrome: assessment of factors associated with transfusion requirements and mortality. J Intensive Care Med. 2017:885066617706339. https://doi.org/ $10.1177 / 0885066617706339$

27. lapichino GE, Protti A, Andreis DT, Panigada M, Artoni A, Novembrino C, et al. Antithrombin during extracorporeal membrane oxygenation in adults: national survey and retrospective analysis. ASAIO J. 2018;65(3):1.

28. Aubron C, DePuydt J, Belon F, Bailey M, Schmidt M, Sheldrake J, et al. Predictive factors of bleeding events in adults undergoing extracorporeal membrane oxygenation. Ann Intensive Care. 2016;6:97.

29. Panigada M, lapichino GE, Brioni M, Panarello G, Protti A, Grasselli G, et al. Thromboelastography-based anticoagulation management during extracorporeal membrane oxygenation: a safety and feasibility pilot study. Ann Intensive Care. 2018:8:1-9.

Ready to submit your research? Choose BMC and benefit from:

- fast, convenient online submission

- thorough peer review by experienced researchers in your field

- rapid publication on acceptance

- support for research data, including large and complex data types

- gold Open Access which fosters wider collaboration and increased citations

- maximum visibility for your research: over $100 \mathrm{M}$ website views per year

At $\mathrm{BMC}$, research is always in progress.

Learn more biomedcentral.com/submission 